\title{
Correção de assimetria gengival através de gengivectomia com osteotomia minimamente traumática
}

Correction of gingival asymmetry using gingivectomy with minimally traumatic osteotomy

Corrección de la asimetría gingival mediante gingivectomía con osteotomía mínimamente traumática

Andressa Nascimento de SOUZA ${ }^{1}$

André Rodrigo Justino da SILVA ${ }^{2}$

Elaine Patrícia Lima SILVA ${ }^{3}$

${ }^{1}$ Residente do Programa de Residência em Atenção Básica pela Escola Multicampi de Ciências Médicas (EMCM), Universidade Federal do Rio Grande do Norte (UFRN), 59300-000, Caicó - RN, Brasil

${ }^{2}$ Mestrando do Programa de Pós-Graduação em Odontologia pela Faculdade de Odontologia de Pernambuco, Universidade de Pernambuco (FOP/UPE), 54756-220, Camaragibe - PE, Brasil

${ }^{3}$ Cirurgiã-Dentista, Pós-graduanda (especialização) em Ortodontia pelo Instituto de Odontologia das Américas,

Núcleo de Estudos e Aperfeiçoamento Odontológico (IOA-NEAO), 58045-120, João Pessoa - PB, Brasil

\section{Resumo}

Introdução: Quando existe desarmonia ou assimetria no conjunto dentes-gengiva-lábios, o status psicológico e social do indivíduo fica prejudicado e se faz necessário alguma intervenção para promover satisfação e bem estar ao paciente. Objetivo: Relatar um caso de correção de assimetria gengival através da técnica de gengivectomia com osteotomia minimamente traumática. Relato de Caso: Paciente de 22 anos, sexo feminino, leucoderma compareceu para atendimento tendo como queixa a assimetria de gengiva no sorriso. Após a avaliação clínica e periodontal viu-se a necessidade da cirurgia de gengivectomia associada a osteotomia minimamente traumática (flapless). Inicialmente realizou-se anestesia terminal infiltrativa utilizando o anestésico mepivacaína com vasoconstrictor, marcação dos pontos através da sondagem utilizando a sonda periodontal de Williams na região vestibular dos dentes $11,12,21$ e 23, após isso a remoção do tecido gengival foi realizada com bisturi $15 \mathrm{c}$ e seguiu-se com a osteotomia através do sulco gengival utilizando o Cinzel Mini-Ochsenbein N². Durante e após o procedimento observou-se a distância entre a crista óssea alveolar e a margem gengival, que deve ser de $3 \mathrm{~mm}$. Concluída a osteotomia fez-se a gengivoplastia com ponta diamantada 3118 em alta rotação. Por fim foi efetuada a irrigação abundante com soro fisiológico; hemostasia; e orientações pós-operatórias, incluindo o uso de Punica granatum Linn (romã) para bochecho. Conclusão: Quando corretamente indicada e executada, a técnica de gengivectomia com osteotomia apenas por via sulcular com uso de cinzel periodontal, sem elevação de retalho é eficaz na correção de assimetrias dos dentes anteriores.

Descritores: Gengivectomia; Osteotomia; Estética Dentária; Sorriso.

\section{Abstract}

Introduction: When there is disharmony or asymmetry in the teeth-gum-lips group, the individual's psychological and social status is impaired and some intervention is necessary to promote patient satisfaction and well-being. Objective: To report a case of correction of gingival asymmetry using the gingivectomy technique with minimally traumatic osteotomy. Case Report: A 22 year-old leucoderma female patient came to the clinic complaining of gingival asymmetry in the smile. After the clinical and periodontal evaluation, the need for gingivectomy surgery associated with minimally traumatic osteotomy (flapless) was seen. Initially, infiltrative terminal anesthesia was performed using the Mepivacaine anesthetic with vasoconstrictor, marking the points through the probe using the Williams periodontal probe in the buccal region of teeth 11, 12, 21 and 23, after which the removal of the gingival tissue was performed with a scalpel $15 \mathrm{c}$ and followed by osteotomy through the gingival sulcus using the MiniOchsenbein Chisel №2. During and after the procedure, the distance between the alveolar bone crest and the gingival margin, which must be $3 \mathrm{~mm}$, was observed. After osteotomy, gingivoplasty was performed with a diamond tip 3118 in high rotation. Finally, abundant irrigation with saline was performed; hemostasis; and postoperative guidelines, including the use of Punica granatum Linn (pomegranate) for mouthwash. Conclusion: When correctly indicated and performed, the gingivectomy technique with osteotomy only through the sulcular route using periodontal chisels, without flap elevation, is effective in correcting asymmetries of the anterior teeth.

Descriptors: Gingivectomy; Osteotomy; Esthetics, Dental; Smiling.

\section{Resumen}

Introducción: cuando hay desarmonía o asimetría en el grupo de dientes, encías y labios, el estado psicológico y social del individuo se ve afectado y es necesaria alguna intervención para promover la satisfacción y el bienestar del paciente. Objetivo: informar un caso de corrección de la asimetría gingival utilizando la técnica de gingivectomía con osteotomía mínimamente traumática. Informe del caso: una paciente de 22 años leucodermia llegó a la clínica quejándose de la asimetría gingival en la sonrisa. Después de la evaluación clínica y periodontal, se observó la necesidad de cirugía de gingivectomía asociada con osteotomía mínimamente traumática (sin colgajo). Inicialmente, la anestesia terminal infiltrativa se realizó con el anestésico de mepivacaína con vasoconstrictor, marcando los puntos a través de la sonda con la sonda periodontal Williams en la región bucal de los dientes $11,12,21$ y 23 , después de lo cual se realizó la extracción del tejido gingival con un bisturí $15 \mathrm{c}$ y seguido de osteotomía a través del surco gingival utilizando el Mini-Ochsenbein Chisel №2. Durante y después del procedimiento, se observó la distancia entre la cresta ósea alveolar y el margen gingival, que debe ser de $3 \mathrm{~mm}$. Después de la osteotomía, la gingivoplastia se realizó con una punta de diamante 3118 en alta rotación. Finalmente, se realizó un riego abundante con solución salina; hemostasia y pautas postoperatorias, incluido el uso de Punica granatum Linn (granada) para enjuague bucal. Conclusión: cuando se indica y realiza correctamente, la técnica de gingivectomía con osteotomía solo a través de la ruta sulcular usando cinceles periodontales, sin elevación del colgajo, es efectiva para corregir las asimetrías de los dientes anteriores.

Descriptores: Gingivectomía; Osteotomía; Estética Dental; Sonrisa.

INTRODUÇÃO

O estabelecimento de uma boa relação
interpessoal atualmente está bastante
relacionado a uma aparência física agradável e
o sorriso faz parte dessa percepção, de modo

que se relaciona à expressão de sentimentos como autoconfiança, simpatia, autocuidado, entre outros. Por outro lado, quando existe desarmonia ou assimetria no conjunto dentes- 
gengiva-lábios o status psicológico e social do indivíduo fica prejudicado, pelo fato de trazer implicações negativas na sua apresentação à sociedade ${ }^{1}$.

Sendo assim, a assimetria se define como falta de proporção e harmonia entre os três elementos do conjunto já citado. Existem fatores essenciais na caracterização de um sorriso atrativo e harmonioso, sendo eles a simetria entre cor e tamanho dos dentes, posição do lábio superior e o grau de exibição gengival ${ }^{2}$.

Quando o paciente, durante o sorriso apresenta exposição gengival superior a $3 \mathrm{~mm}$ ele é caracterizado como sorriso gengival. A etiologia desta condição pode estar relacionada a situações como extrusão dentoalveolar anterior, erupção passiva alterada, crescimento vertical excessivo da maxila, lábio curto, hiperatividade do lábio superior e hiperplasia gengival ${ }^{3}$.

Desse modo, após o estabelecimento da etiologia, o profissional possui várias opções de tratamentos que se mostram eficaz tanto na medicina estética quanto na odontologia, podendo ser feita associação de técnicas para atingir um resultado final satisfatório. Uma opção bastante escolhida é a cirurgia periodontal que devolve a anatomia dentogengival e promove satisfação e bemestar aos pacientes ${ }^{4}$. A técnica de gengivectomia e osteotomia minimamente invasiva consegue reestabelecer a harmonia do sorriso, por se mostrar uma excelente conduta para os casos de exposição gengival excessiva ao sorrir e de contorno inadequado da margem gengival ${ }^{5}$.

O tratamento cirúrgico é considerado minimamente invasivo quando não envolve levantamento de retalho e ainda assim apresenta resultados estéticos e funcionais tão eficientes quanto às técnicas convencionais ${ }^{6}$. A técnica minimamente traumática pode ser também chamada de flapless, na qual a osteotomia é feita pelo sulco gengival com o auxílio de microcinzeis e suas vantagens são o menor tempo cirúrgico, resultados previsíveis e esteticamente favoráveis desde que realizada seguindo todos os passos do protocolo e com indicação adequada, que inclui erupção passiva alterada como fator etiológico, biótipo ósseo fino ou intermediário e tecido queratinizado abundante ${ }^{7}$.

O objetivo do presente estudo é relatar um caso de correção de assimetria gengival no qual a técnica de gengivectomia com osteotomia minimamente traumática foi utilizada.

\section{CASO CLÍNICO}

Paciente leucoderma, 22 anos, sexo feminino, sem alterações sistêmicas, com saúde periodontal e ausência de hiperplasia gengival, procurou atendimento com queixa principal de sorriso gengival e assimétrico, com histórico de uso de aparelho ortodôntico (Figura 1).

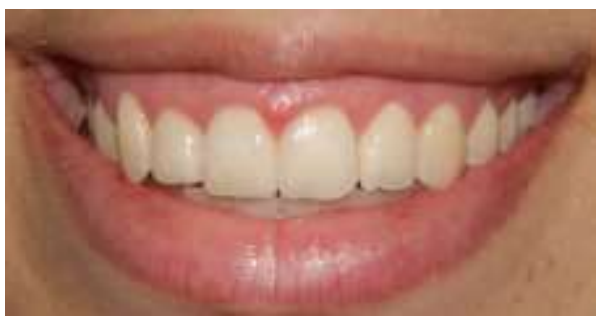

Figura 1: Aspecto clínico inicial: observa-se excesso de exposição gengival e assimetria no contorno.

Os exames periodontais constataram uma gengiva saudável em margem, contorno, coloração e textura, com profundidade de sondagem entre $1,5 \mathrm{~mm}$ e $2 \mathrm{~mm}$, mucosa ceratinizada de $7 \mathrm{~mm}$ a $9 \mathrm{~mm}$ e gengiva inserida entre $5,5 \mathrm{~mm}$ e $7 \mathrm{~mm}$, a paciente apresentava erupção passiva alterada nos dentes 12 e 23. Optou-se pelo aumento de coroa clínica, através de gengivectomia em bisel interno com osteotomia pela técnica fechada nos elementos $11,12,21$ e 23, com a finalidade de melhorar o contorno e a simetria gengival.

Após a antissepsia extra e intrabucal, realizou-se anestesia infiltrativa em fundo de sulco com Mepivacaína a 3\% com vasoconstritor (DFL- Rio de Janeiro, Rio de Janeiro, Brasil). Com o auxílio de uma Sonda Periodontal Milimetrada 15mm (Golgran- São Caetano do Sul, São Paulo, Brasil), foram demarcados os pontos sangrantes que correspondem aos zênites desejados dos elementos 11, 12, 21 e 23. A partir disso, foi feita a remoção do tecido gengival com lâmina de bisturi $\mathrm{n}$ - $15 \mathrm{C}$ nos elementos acima citados, sem remoção das papilas gengivais (Figura 2).

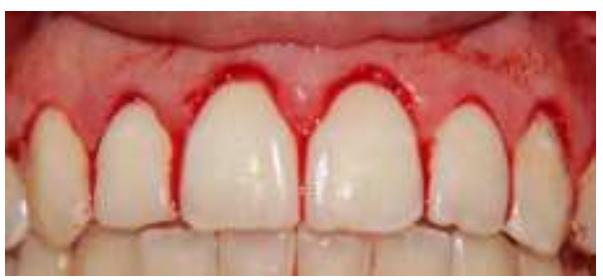

Figura 2: Aspecto pós- gengivectomia.

Em seguida, realizou-se a osteotomia pela técnica minimamente invasiva, utilizando Cinzel Mini-Ochsenbein N ${ }^{\circ} 2$ (Fava- Franco da Rocha, São Paulo, Brasil) na qual a ponta ativa desse instrumento foi introduzida no sulco gengival vestibular dos elementos dentais, inserindo-a sob o osso da crista alveolar e realizando movimentos leves a fim de remover quantidade suficiente de osso. Durante e após a 
osteotomia, ocorreu a avaliação da distância entre a crista óssea alveolar e a margem gengival, que deve ser de $3 \mathrm{~mm}$ para que não haja recidiva no encurtamento da coroa clínica dos elementos corrigidos (Figura 3). Após a osteotomia, procedeu-se à raspagem e alisamento corono-radicular para a remoção dos fragmentos ósseos e posterior irrigação com soro fisiológico. Posteriormente, foi feita a gengivoplastia com broca diamantada 3118 (Microdont- Socorro, São Paulo, Brasil) em alta rotação, sob irrigação constante com solução de cloreto de sódio a 0,9\% (Figura 4). Foi prescrito bochechos com extrato de Punica granatum Linn (romã) e Dipirona Sódica $500 \mathrm{mg}$ em caso de dor, este último, porém, não foi administrado pela paciente que relatou não ter sentido dor no pós-operatório.

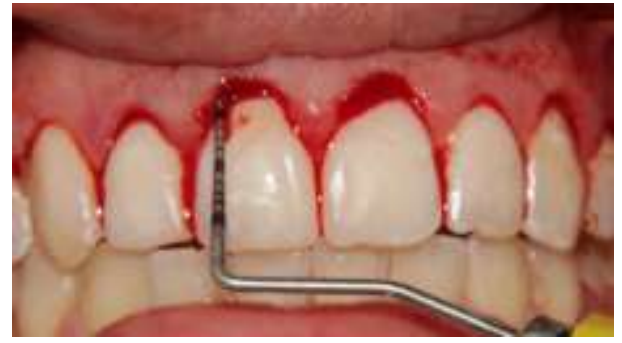

Figura 3: Sondagem para avaliação da profundidade durante e após a osteotomia.



Figura 4: Gengivoplastia com ponta diamantada 3118 em alta rotação.

No pós-operatório de 21 dias, a paciente foi examinada e se observou saúde periodontal, bem como melhora na simetria e no contorno gengival, proporcionando um sorriso mais harmonioso (Figura 5).

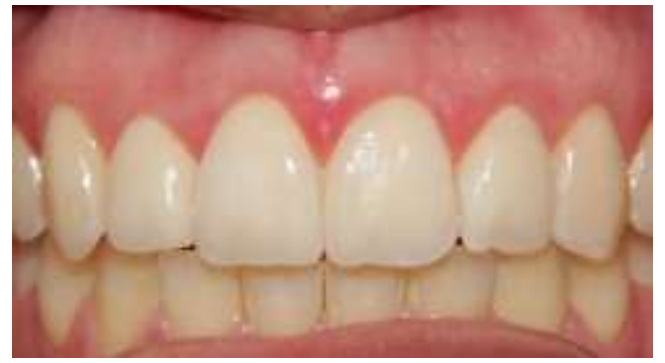

Figura 5: Aspecto pós-operatório de 21 dias: vê-se melhora na harmonia do sorriso da paciente.

DISCUSSÃO

O procedimento cirúrgico de aumento de coroa clínica é bastante eleito para correção de sorriso gengival, recuperando 0 espaço biológico através da remoção de tecidos periodontais para exposição de coroa clínica acima da crista óssea alveolar ${ }^{8}$. Um estudo de Gargiulo et al. $^{9}$ com animais propõe que a medida ideal após sondagem transulcular é de um valor médio de $3 \mathrm{~mm}$ para recuperação dos tecidos supracrestais.

Desse modo, o procedimento de aumento de coroa clínica pode se caracterizar somente como gengivectomia (apenas com remoção de tecido gengival) e/ou técnicas com retalho, envolvendo ou não osteotomia podendo ser realizado tanto em dentes com cáries e restaurações subgengivais quanto em casos estéticos ${ }^{4,9}$, como realizado no presente estudo.

Para fins de diagnóstico e escolha de tratamento, 0 excesso gengival pode ser classificado em: tipo I, com a junção mucogengival apical à crista óssea e tipo II, com junção mucogengival ao nível ou em posição coronal em relação à crista óssea; subtipo $A$, com pelo menos $2 \mathrm{~mm}$ entre a junção amelocementária e a crista óssea e subtipo $B$, com menos de $2 \mathrm{~mm}$ entre a junção amelocementária e a crista óssea. A partir disso é feita a indicação cirúrgica $=$ tipo I $A$ : gengivoplastia ou gengivectomia; tipo I B: retalho mucoperiosteal com remoção da margem gengival e osteotomia; tipo II A: retalho de espessura parcial deslocado apicalmente; tipo II B: retalho de espessura total reposicionado apicalmente com osteotomia ${ }^{10,11}$.

No caso relatado no presente estudo foi tomada a escolha pela realização da técnica de gengivectomia minimamente traumática tendo como base as vantagens encontradas na literatura que apontam mínimo trauma aos tecidos periodontais e dentais ao passo que aperfeiçoa a saúde estética do sorriso ${ }^{7,12}$, além de apresentar maior previsibilidade de resultados. Lobo et al. ${ }^{7}$ relatam mínima lesão aos tecidos e vasos sanguíneos, o que leva a uma recuperação mais branda e uniforme, redução da morbidade pós-operatória e ao aumento do conforto do paciente. Por outro lado, a técnica flapless apresenta a limitação de depender muito da habilidade do operador para localizar através do sulco gengival a crista óssea em relação à junção amelocementária, portanto exige-se que a execução da técnica seja feita por um profissional experiente ${ }^{7}$.

Muitos estudos têm destacado a Punica granatum Linn (romã) como agente eficaz para cicatrização pós-operatória, pois essa planta tem evidenciado resultados positivos no controle do biofilme dental e reparo tecidual. Em 2015, Nóbrega et al. ${ }^{13}$ avaliaram um enxaguatório bucal contendo Punica granatum L. (romã), com 
- objetivo de estudar a eficácia clínica no controle do biofilme dental e inflamação gengival em comparação com o digluconato de clorexidina a 0,12\%. O Índice de Placa (IP) e o Índice de sangramento Gengival (ISG) foram usados para avaliar o controle do biofilme e inflamação gengival. Os resultados mostraram que os bochechos de romã reduziram os valores médios do IP e ISG, mas sem diferença significativa. No entanto, houve uma redução significativa na contagem de Streptococcus orais.

Desse modo, assim como no presente trabalho, Pontes et al. ${ }^{6}$ demonstraram que 0 aumento de coroa através da técnica minimamente invasiva é capaz de melhorar o sorriso gengival e estabelecer um contorno gengival harmônico e simétrico, expondo de modo mais esteticamente agradável as coroas clínicas.

\section{CONCLUSÃO}

A partir da avaliação clínica pode-se perceber que, quando corretamente indicada $e$ executada a técnica de gengivectomia com osteotomia apenas por via sulcular com uso de cinzel periodontal, sem elevação de retalho é eficaz na correção de assimetrias dos dentes anteriores, devolvendo harmonia para o sorriso e consequentemente melhoria na autoestima do paciente.

\section{REFERÊNCIAS}

1. Mostafa DA. Successful management of sever gummy smile using gingivectomy and botulinum toxin injection: a case report. Int J Surg Case Rep. 2017;42:169-74.

2. Oliveira SAR, Venturim RTZ. Cirurgia Periodontal Ressectiva valorizando o sorriso gengival: relato de caso clínico. Colloq Vitae. 2012;4(2):118-28.

3. Senise IR, Marson FC, Progiante PS, Oliveira e silva C. O uso de toxina botulínica como alternativa para 0 tratamento do sorriso gengival causado pela hiperatividade do lábio superior. Uningá Rev. 2015;23 (3):104-10.

4. Trentini MS, Bittencourt ME, Carli JP, Sachetti DG, Durigon M. Correção de assimetria dentogengival com finalidade estética: Relato de caso clínico. Salusvita. 2018;37(3):671-84.

5. Cristóvam AVS, Medeiros JDS, Cruz JHA, Brito RMLV, Figueiredo KA, Rodrigues RQF et al. Correção de contorno gengival pelas técnicas de gengivectomia convencional e minimamente invasiva. Arch Health Invest. 2019;8(10): 606-12.

6. Pontes SA, Duarte PM, Oliveira ACG, Coelho $\mathrm{EF}$, Esteves FM, Mello GBR et al. Aumento de coroa clínica estético minimamente invasivo: relato de caso de 12 meses. Rev Saúde UNG SER. 2016;10(3/4):55-64.

7. Lobo NS, Wanderley VA, Alves RV. Cirurgia periodontal de aumento de coroa clínica estética sem a elevação do retalho (flapless): relato de caso clínico. Arq Bras Odontol. 2017; 13(1):118-23.

8. Rissato M, Trentin MS. Aumento de coroa clínica para restabelecimento das distâncias biológicas com finalidade restauradora: revisão da Literatura. RFO. 2012;17(2):234-39.

9. Gargiulo AW, Wentz FM, Orban B. Dimensions and relations of the dentogingival junction in human. J Periodontol. 1961;32(3):261-67.

10. Levine RA, McGuire $M$. The diagnosis and treatment of the gummy smile. Compend Contin Educ Dent. 1997;18(8):757-62, 764; quiz 766.

11.Sousa SJB, Magalhães D, Silva GR, Soares CJ, Soares PFB, Santos-filho PCF. Cirurgia plástica periodontal para correção de sorriso gengival associada à restaurações em resina composta: relato de caso clínico. ROBRAC. 2010;19(51):362-66.

12. Ribeiro FV, Hirata DY, Reis AF, Santos VR, Miranda TS, Faveri $M$ et al. Open-flap versus flapless esthetic crown lengthning: 12-month clinical outcomes of a randomized controlled clinical trial. J Periodontol. 2014;85(4):536-44.

13. Nóbrega DRM, Santos RL, Soares RSC, Alves PM, Medeiros ACD, Pereira JV. A randomized, controlled clinical trial on the clinical and microbiological efficacy of Punica granatum Linn mouthwash. BRPDIC. 2015;15(1):301-8.

\section{CONFLITO DE INTERESSES}

Os autores declaram não haver conflitos de interesse

\section{AUTOR PARA CORRESPONDÊNCIA}

\section{André Rodrigo Justino da Silva}

Instituto de Tecnologia de Pernambuco - ITEP/

Bloco B - 1aaar

Faculdade de Odontologia da Universidade de Pernambuco

Av. Prof. Luis Freire, 700 - Cidade Universitária 50740-540 Recife-PE, Brasil

E-mail: andrerodonto@gmail.com 